CAU-THP-95-38

CERN-TH/96-67

DESY 96-038

HD-THEP-96-05

cond-mat/9603129

\title{
Flow of the Coarse Grained Free Energy for Crossover Phenomena
}

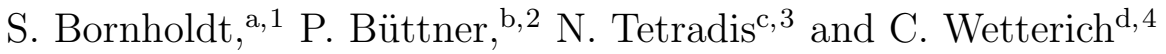 \\ ${ }^{a}$ Institut für Theoretische Physik, Universität Kiel, \\ Leibnizstr. 15, 24098 Kiel, Germany \\ bII. Institut für Theoretische Physik, Universität Hamburg, \\ Luruper Chaussee 149, 22761 Hamburg, Germany \\ ${ }^{\mathrm{C}} \mathrm{CERN}$, Theory Division, \\ CH-1211, Geneva 23, Switzerland \\ dInstitut für Theoretische Physik, Universität Heidelberg, \\ Philosophenweg 16, 69120 Heidelberg, Germany
}

\begin{abstract}
The critical behaviour of a system of two coupled scalar fields in three dimensions is studied within the formalism of the effective average action. The fixed points of the system are identified and the crossover between them is described in detail. Besides the universal critical behaviour, the flow of the coarse grained free energy also describes the approach to scaling.
\end{abstract}

\footnotetext{
${ }^{1}$ e-mail: bornholdt@theo-physik.uni-kiel.de

2e-mail: pesebu@x4u2.desy.de

${ }^{3}$ e-mail: tetradis@surya11.cern.ch

${ }^{4}$ e-mail: c.wetterich@thphys.uni-heidelberg.de
} 


\section{Introduction}

The properties of multicomponent statistical systems near the critical temperature of a second-order phase transition are often characterized by a crossover behaviour. Crossover typically occurs if two or more fixed points are relevant for the description of scaling. Examples can be found in physical systems with small impurities or a small coupling between two degrees of freedom. A well developed formalism for the description of the universal features of critical crossover is based on the renormalization group equations [1]-[3]. Nevertheless, the practical situation in a given experiment may be quite complicated. The correlation length is large but not infinite in real life and, as a consequence, the scaling properties are not perfect. The temperature dependence of order parameters, susceptibilities, etc., often reflects an interplay between the approach to scaling and crossover. The first is determined by the flow of couplings towards a fixed point, whereas the second corresponds to the running of couplings from one fixed point to another. Standard methods employed for the study of critical phenomena, such as for example the $\epsilon$-expansion, are often insufficient for the quantitative description of such complex situations, which are in addition not completely governed by universal scaling behaviour. Numerical simulations are also difficult in this context, as typically several different length scales are involved.

We propose here the use of a field theoretical description in terms of the effective average action $\Gamma_{k}$. This corresponds to a coarse grained free energy where fluctuations with momenta larger than $k$ are integrated out. The flow of $\Gamma_{k}$ with a change of the infrared cutoff scale $k$ is described by an exact non-perturbative flow equation. This can be solved approximately by appropriate truncations of the most general form of $\Gamma_{k}$. At a given scale $k$ only modes with momenta around $k$ contribute to the flow. We specify the microscopic physics by the "initial value" of $\Gamma_{k}$ at some high momentum scale $\Lambda$. For a given experimental situation this step involves the rewriting of the problem in terms of continuous variables. This part of the problem is not universal and involves only the short distance modes. It is not treated in the present paper. We only mention here that, for lattice models, it is advantageous to take $\Lambda$ somewhat smaller than the inverse lattice spacing, so that deviations of the form of $\Gamma_{\Lambda}$ from translational and rotational invariance are not too large. Our formalism can be extended to situations without translational and rotational symmetry. It is, however, much simpler for translationally and rotationally invariant situations where standard field theory can be used. In the vicinity of the critical temperature $T_{c r}$ of a second-order phase transition one of the couplings parametrizing $\Gamma_{\Lambda}$ - in our case $\delta \kappa_{\Lambda}$ - can be used to describe the distance from the critical hypersurface in the space of couplings, i.e. $\delta \kappa_{\Lambda} \sim T-T_{c r}$. The order parameter, correlation lengths etc. can then be studied as functions of $\delta \kappa_{\Lambda}$. For crossover situations there exists a second important coupling - in our case $x_{\Lambda}$ - which determines to which fixed point the flow of the couplings is dominantly attracted. The parameter $x_{\Lambda}$ typically measures the amount of impurities or the size of a coupling between different components of a system. Critical quantities, such as the exponent $\nu$ which governs the dependence of the correlation length on $\delta \kappa_{\Lambda}\left(\nu=-\partial \ln \xi / \partial \ln \left|\delta \kappa_{\Lambda}\right|\right)$, now depend on the ratio $x_{\Lambda} /\left|\delta \kappa_{\Lambda}\right|$.

We determine the long distance physics by solving numerically the flow of $\Gamma_{k}$, starting 
at short distances with $\Gamma_{\Lambda}$ and moving towards $k=0$. For $k=0$ the effective average action equals the free energy expressed as a functional of the fields. In the language of field theory, $\Gamma_{0}$ is the generating functional of the 1PI Green functions, and all physically relevant information can be extracted from it. The method is formulated in arbitrary dimensions and can, in particular, be applied directly to three-dimensional statistical systems. Consequently, there is no restriction to the scaling situation for infinitesimally small $\delta \kappa_{\Lambda}$. The correlation length and similar quantities can be computed for arbitrary $\delta \kappa_{\Lambda}$ and $x_{\Lambda}$, and the interplay between non-universal and universal behaviour can be studied by approaching the critical temperature $\left(\delta \kappa_{\Lambda} \rightarrow 0\right)$.

In this paper we discuss these ideas for a specific model of two coupled fields with discrete symmetry. The parameter $x_{\Lambda}$ will be a measure of the strength of the coupling between the two fields. For the present investigation our main purpose is not high accuracy for the universal quantities but rather a demonstration that numerical solutions of flow equations can give a realistic picture. We, therefore, limit ourselves to a relatively rough truncation of $\Gamma_{k}$. More involved truncations would give better quantitative accuracy but also obscure the presentation of the basic mechanisms and complicate the numerical work. Such improvements will become relevant once a given experimental setting with given form of $\Gamma_{\Lambda}$ is investigated, with emphasis on quantitative accuracy. They are also needed if one attempts to compute universal quantities such as crossover exponents with an accuracy comparable with other existing methods.

\section{The evolution equation for the potential}

We consider a theory of two real scalar fields $\chi_{a}(a=1,2)$, in $d$-dimensional Euclidean space, with an action $S[\chi]$ invariant under the discrete symmetry $(1 \leftrightarrow-1,2 \leftrightarrow-2,1 \leftrightarrow$ 2 ). We specify the action together with some ultraviolet momentum cutoff $\Lambda$, so that the theory is properly regulated. We add to the kinetic term an infrared regulating piece [四]

$$
\Delta S=\frac{1}{2} \int \frac{d^{d} q}{(2 \pi)^{d}} R_{k}(q) \chi_{a}^{*}(q) \chi^{a}(q)
$$

where $\chi^{a}(q)$ are the Fourier modes of the scalar fields. The function $R_{k}$ is employed in order to prevent the propagation of modes with characteristic momenta $q^{2}<k^{2}$. This can be achieved, for example, by the choice

$$
R_{k}(q)=\frac{Z_{k} q^{2} f_{k}^{2}(q)}{1-f_{k}^{2}(q)}, \quad \text { with } \quad f_{k}^{2}(q)=\exp \left(-\frac{q^{2}}{k^{2}}\right) .
$$

The quantity $Z_{k}$ is an appropriate wave function renormalization whose precise definition will be given below. The modes with $q^{2} \gg k^{2}$ are unaffected by the infrared cutoff, while the low frequency modes with $q^{2} \ll k^{2}$ are cut off, as $R_{k}$ acts like a mass term: $\lim _{q^{2} \rightarrow 0} R_{k}(q)=Z_{k} k^{2}$. Through the usual Legendre transformation we obtain the generating functional for the 1PI Green functions $\tilde{\Gamma}_{k}\left[\phi^{a}\right]$, where $\phi^{a}$ is the expectation value of the field $\chi^{a}$ in the presence of sources. The use of the modified propagator for the calculation 
of $\tilde{\Gamma}_{k}$ results in the effective integration of only the fluctuations with $q^{2}>k^{2}$. Finally, the effective average action is obtained by subtracting the infrared cutoff piece

$$
\Gamma_{k}\left[\phi^{a}\right]=\tilde{\Gamma}_{k}\left[\phi^{a}\right]-\frac{1}{2} \int \frac{d^{d} q}{(2 \pi)^{d}} R_{k}(q) \phi_{a}^{*}(q) \phi^{a}(q) .
$$

For $k$ equal to the ultraviolet cutoff $\Lambda, \Gamma_{k}$ becomes equal to the classical action $S$ (no effective integration of modes takes place), while for $k \rightarrow 0$ it tends towards the effective action $\Gamma$ (all the modes are included), which is the generating functional of the 1PI Green functions computed from $S$ without infrared cutoff. For intermediate values of $k$ the effective average action realizes the concept of a coarse grained free energy [5].

The interpolation of $\Gamma_{k}$ between the classical and the effective action makes it a very useful field theoretical tool. The means for practical calculations is provided by an exact flow equation, which describes the response of the effective average action to variations of the infrared cutoff $(t=\ln (k / \Lambda))$ 进

$$
\frac{\partial}{\partial t} \Gamma_{k}[\phi]=\frac{1}{2} \operatorname{Tr}\left\{\left(\Gamma_{k}^{(2)}[\phi]+R_{k}\right)^{-1} \frac{\partial}{\partial t} R_{k}\right\} .
$$

Here $\Gamma_{k}^{(2)}$ is the second functional derivative of the effective average action with respect to $\phi^{a}$.

For the solution of eq. (2.4) an efficient truncation scheme has to be developed. The form of the effective average action is constrained by the $(1 \leftrightarrow-1,2 \leftrightarrow-2,1 \leftrightarrow$ 2) symmetry. However, there is still an infinite number of invariants to be considered. Throughout this paper we work with an approximation which keeps only a standard kinetic term in the effective average action

$$
\Gamma_{k}=\int d^{d} x\left\{U_{k}\left(\rho_{1}, \rho_{2}\right)+\frac{1}{2} Z_{k} \partial^{\mu} \phi_{a} \partial_{\mu} \phi^{a}\right\}
$$

and neglect all invariants which involve more derivatives of the fields. We have used the definition $\rho_{a}=\frac{1}{2} \phi_{a}^{2}$. The parameter appearing in eq. (2.2) can be identified with the wave function renormalization $Z_{k}$. The justification for our approximation lies in the smallness of the anomalous dimension, which is expected to be $\eta \simeq 0.04$ for the three-dimensional theory. We estimate the corrections arising from the inclusion of higher derivative terms to be of the same order as $\eta$. In order to obtain an evolution equation for the effective average potential $U_{k}$ from eq. (2.4), we have to expand around a constant background field configuration (so that the derivative terms in the parametrization (2.5) do not contribute to the 1.h.s. of eq. (2.4)). Equation (2.4) then gives [6, 7]

$$
\frac{\partial}{\partial t} U_{k}\left(\rho_{1}, \rho_{2}\right)=\frac{1}{2} \int \frac{d^{d} q}{(2 \pi)^{d}}\left(\frac{1}{P\left(q^{2}\right)+M_{1}^{2}}+\frac{1}{P\left(q^{2}\right)+M_{2}^{2}}\right) \frac{\partial}{\partial t} R_{k}(q) .
$$

$P\left(q^{2}\right)$ results from the combination of the standard kinetic contribution $Z_{k} q^{2}$ and the regulating term $R_{k}$ into an effective inverse propagator (for massless fields)

$$
P\left(q^{2}\right)=Z_{k} q^{2}+R_{k}=\frac{Z_{k} q^{2}}{1-f_{k}^{2}(q)}
$$


with $f_{k}^{2}(q)$ given in eq. $(2.2) ; M_{1,2}^{2}$ are the eigenvalues of the mass matrix at the point $\left(\rho_{1}, \rho_{2}\right)[8,9]:$

$$
M_{1,2}^{2}=\frac{1}{2}\left\{U_{1}+U_{2}+2 U_{11} \rho_{1}+2 U_{22} \rho_{2} \pm\left[\left(U_{1}-U_{2}+2 U_{11} \rho_{1}-2 U_{22} \rho_{2}\right)^{2}+16 U_{12}^{2} \rho_{1} \rho_{2}\right]^{\frac{1}{2}}\right\},
$$

and we have introduced the notation $U_{1}=\partial U_{k} / \partial \rho_{1}, U_{12}=\partial^{2} U_{k} / \partial \rho_{1} \partial \rho_{2}$, etc.

The fixed point structure of the theory is more easily identified if we use the dimensionless renormalized parameters

$$
\begin{aligned}
\tilde{\rho}_{a} & =Z_{k} k^{2-d} \rho_{a} \\
u_{k}\left(\tilde{\rho}_{1}, \tilde{\rho}_{2}\right) & =k^{-d} U_{k}\left(\rho_{1}, \rho_{2}\right) \\
\tilde{m}_{a}^{2} & =Z_{k}^{-1} k^{-2} M_{a}^{2} .
\end{aligned}
$$

The rescaled mass eigenvalues $\tilde{m}_{1,2}^{2}$ are related to $u_{k}, \tilde{\rho}_{1}, \tilde{\rho}_{2}$ through expressions analogous to eqs. (2.8), with $u_{1}=\partial u_{k} / \partial \tilde{\rho}_{1}$, etc. The evolution equation (2.6) can now be written in the scale-independent form

$$
\frac{\partial}{\partial t} u\left(\tilde{\rho}_{1}, \tilde{\rho}_{2}\right)=-d u+(d-2+\eta)\left(\tilde{\rho}_{1} u_{1}+\tilde{\rho}_{2} u_{2}\right)-v_{d} L_{0}^{d}\left(\tilde{m}_{1}^{2}\right)-v_{d} L_{0}^{d}\left(\tilde{m}_{2}^{2}\right),
$$

with

$$
v_{d}^{-1}=2^{d+1} \pi^{\frac{d}{2}} \Gamma\left(\frac{d}{2}\right) .
$$

The functions $L_{0}^{d}(w)$ are given by

$$
L_{0}^{d}(w)=-2 \int_{0}^{\infty} d y y^{\frac{d}{2}+1} \frac{\exp (-y)}{[1-\exp (-y)]^{2}}\left[\frac{y}{1-\exp (-y)}+w\right]^{-1} .
$$

We also employ the functions

$$
\begin{array}{ll}
L_{1}^{d}(w)=-\frac{\partial}{\partial w} L_{0}^{d}(w) & \\
L_{n+1}^{d}(w)=-\frac{1}{n} \frac{\partial}{\partial w} L_{n}^{d}(w) & \text { for } n \geq 1
\end{array}
$$

in the following. These functions are negative for all values of $w$. Also $\left|L_{n}^{d}(w)\right|$ are monotonically decreasing for increasing $w$ and introduce threshold behaviour in the evolution. For large values of $\tilde{m}_{a}^{2} \sim M_{a}^{2} / k^{2}$ the last two terms in eq. (2.10) vanish and the evolution of $U_{k}$ stops. The above functions have been extensively discussed in refs. [6, 7, 15]. As they have no simple analytical form, we use numerical fits in the following.

The evolution of the wave function renormalization $Z_{k}$, which gives the anomalous dimension

$$
\partial\left(\ln Z_{k}\right) / \partial t=-\eta
$$


can be computed from the exact flow equation (2.4) by expanding around a background field configuration with a small momentum dependence [7]. We work in three dimensions and use the approximate value $\eta=0.04$. Within the accuracy of our results, this value is consistent with the values of $\eta$ in both the Ising and Heisenberg models, which are relevant near the fixed points. We take into account threshold effects in the evolution of $Z_{k}$ and the vanishing of $\eta$ in the symmetric regime (when the minimum of $U_{k}$ is at the origin) [7] by using $\eta=0.04$ as long as the theory is in the regime with spontaneous symmetry breaking and the mass eigenvalues are in the interval $0.01 \leq \tilde{m}_{a}^{2} \leq 100$. Otherwise we set $\eta=0$. We also point out that we have neglected a term proportional to $\eta$ in the r.h.s. of (2.10), which comes from the $t$-derivative acting on $Z_{k}$ inside $R_{k}$ in the r.h.s. of eq. (2.6). This is consistent with our approximate treatment of the wave function renormalization effects and is justified by the smallness of $\eta$.

\section{The fixed point structure}

From this point on we concentrate on the three-dimensional theory. Equation (2.10), with $d=3$, is the master equation for our investigation. It is a non-linear partial differential equation for three independent variables. A numerical solution is possible (see refs. [10, 11] for the solution of the evolution equation for the $O(N)$-symmetric scalar theory). However, the presence of three independent variables complicates the numerical treatment. For this reason we resort to a different approximation scheme. We first introduce a Taylor expansion of $U_{k}\left(\rho_{1}, \rho_{2}\right)$ around a certain point (usually the minimum of the potential or the origin). This turns eq. (2.6) into an infinite system of ordinary (coupled) differential equations for the $k$-dependence of the point around which the expansion is performed and the derivatives of the potential, with independent variable $t=\ln (k / \Lambda)$. We solve this system approximately by truncating at a finite number of derivatives. This approach has been used in the past for the study of the $O(N)$-symmetric scalar theory. It has provided a full, detailed picture of the high-temperature phase transition for the four-dimensional theory [12, 7, 13, 14, with accurate determination (at the few $\%$ level) of such non-trivial quantities as the critical exponents [7]. An estimate [16] of the residual errors for high level truncations indicates that they are smaller than the uncertainties introduced by the imprecise treatment of the wave function renormalization effects. This has been confirmed by comparison with the numerical solution of the untruncated evolution equation for the potential [10, 11]. The same approach has been used in ref. [9] for the study of the high-temperature phase transition in a four-dimensional theory of two scalars with a $(1 \leftrightarrow-1,2 \leftrightarrow-2,1 \leftrightarrow 2)$ symmetry. The truncation employed there kept up to second derivatives of the potential $U_{11}, U_{12}, U_{22}$. The present work is a detailed investigation of the crossover phenomena in the effective three-dimensional theory near the critical temperature. We use an extended truncation which keeps up to the third derivatives of the potential $U_{111}, U_{122}$, etc. We also note that this theory belongs in the same universality class as the statistical two-component spin systems with cubic anisotropy, whose universal behaviour has been studied in [17]-19. 
Let us first parametrize the potential by its derivatives at the origin ( $\mathrm{S}$ regime)

$$
\begin{aligned}
\bar{m}^{2}(k) & =U_{1}(0)=U_{2}(0) & & \\
\bar{\lambda}(k) & =U_{11}(0)=U_{22}(0) & & \bar{g}(k)=U_{12}(0) \\
\bar{\nu}_{A}(k) & =U_{111}(0)=U_{222}(0) & & \bar{\nu}_{B}(k)=U_{112}(0)=U_{122}(0),
\end{aligned}
$$

where the equality of derivatives is imposed by the discete symmetry of the theory. Our approximation amounts to using, for the potential, the ansatz

$$
U_{k}\left(\rho_{1}, \rho_{2}\right)=\bar{m}^{2}\left(\rho_{1}+\rho_{2}\right)+\frac{\bar{\lambda}}{2}\left(\rho_{1}^{2}+\rho_{2}^{2}\right)+\bar{g} \rho_{1} \rho_{2}+\frac{\bar{\nu}_{A}}{6}\left(\rho_{1}^{3}+\rho_{2}^{3}\right)+\frac{\bar{\nu}_{B}}{2}\left(\rho_{1}^{2} \rho_{2}+\rho_{1} \rho_{2}^{2}\right) .
$$

Evolution equations for the above parameters are obtained by taking derivatives of eq. (2.6) with respect to $\rho_{1,2}$. It is convenient to define the dimensionless couplings

$$
\begin{array}{rlrl}
\tilde{m}^{2}(k) & =Z_{k}^{-1} k^{-2} \bar{m}^{2}(k)=k^{-2} m^{2}(k) & & \\
\tilde{\lambda}(k) & =Z_{k}^{-2} k^{-1} \bar{\lambda}(k)=k^{-1} \lambda(k) & \tilde{g}(k) & =Z_{k}^{-2} k^{-1} \bar{g}(k)=k^{-1} g(k) \\
\nu_{A}(k) & =Z_{k}^{-3} \bar{\nu}_{A}(k) & \nu_{B}(k)=Z_{k}^{-3} \bar{\nu}_{B}(k)
\end{array}
$$

The dimensionless potential $u_{k}\left(\tilde{\rho}_{1}, \tilde{\rho}_{2}\right)$ defined in eqs. (2.9) can be expressed in complete analogy to eq. (3.2) in terms of these dimensionless quantities. The evolution equations for the above quantites have a scale-invariant form, in the sense that the r.h.s. does not explicitly involve a dependence on $k$

$$
\begin{aligned}
\frac{d \tilde{m}^{2}}{d t} & =(-2+\eta) \tilde{m}^{2}+v_{3}(3 \tilde{\lambda}+\tilde{g}) L_{1}^{3}\left(\tilde{m}^{2}\right) \\
\frac{d \tilde{\lambda}}{d t} & =(-1+2 \eta) \tilde{\lambda}-v_{3}\left(9 \tilde{\lambda}^{2}+\tilde{g}^{2}\right) L_{2}^{3}\left(\tilde{m}^{2}\right)+v_{3}\left(5 \nu_{A}+\nu_{B}\right) L_{1}^{3}\left(\tilde{m}^{2}\right) \\
\frac{d \tilde{g}}{d t} & =(-1+2 \eta) \tilde{g}-v_{3}\left(6 \tilde{\lambda} \tilde{g}+4 \tilde{g}^{2}\right) L_{2}^{3}\left(\tilde{m}^{2}\right)+6 v_{3} \nu_{B} L_{1}^{3}\left(\tilde{m}^{2}\right) \\
\frac{d \nu_{A}}{d t} & =3 \eta \nu_{A}+2 v_{3}\left(27 \tilde{\lambda}^{3}+\tilde{g}^{3}\right) L_{3}^{3}\left(\tilde{m}^{2}\right)-3 v_{3}\left(15 \tilde{\lambda} \nu_{A}+\tilde{g} \nu_{B}\right) L_{2}^{3}\left(\tilde{m}^{2}\right) \\
\frac{d \nu_{B}}{d t} & =3 \eta \nu_{B}+2 v_{3}\left(9 \tilde{\lambda}^{2} \tilde{g}+15 \tilde{\lambda} \tilde{g}^{2}+4 \tilde{g}^{3}\right) L_{3}^{3}\left(\tilde{m}^{2}\right)-v_{3}\left(21 \tilde{\lambda} \nu_{B}+5 \tilde{g} \nu_{A}+22 \tilde{g} \nu_{B}\right) L_{2}^{3}\left(\tilde{m}^{2}\right)
\end{aligned}
$$

where $v_{3}=1 / 8 \pi^{2}$. They automatically preserve the discrete symmetry.

We are interested in the fixed points of the last set of equations. First there is the ultraviolet attractive Gaussian fixed point $(\mathrm{G})$ with $\tilde{m}^{2}, \tilde{\lambda}, \tilde{g}, \nu_{A}, \nu_{B}=0$. There are also three fixed points with at least one infrared attractive direction [17] 19. They all appear for $\tilde{m}^{2}<0, \tilde{\lambda}, \tilde{g}, \nu_{A}, \nu_{B} \geq 0$, which means that the minimum of $u_{k}\left(\tilde{\rho}_{1}, \tilde{\rho}_{2}\right)$ is away from the origin. (The exact values are not important since the discussion in this section concentrates on the qualitative behaviour only.) For their identification we use their standard 
names in statistical physics [17]-[19]:

a) The Heisenberg fixed point $(\mathrm{H})$ has $\tilde{g}=\tilde{\lambda}, \nu_{B}=\nu_{A}$ and corresponds to a theory with symmetry increased to $O(2)$. The potential $u_{k}\left(\tilde{\rho}_{1}, \tilde{\rho}_{2}\right)$ has a series of degenerate minima at $\tilde{\rho}_{10}+\tilde{\rho}_{20}=\tilde{\rho}_{0}$.

b) The Ising fixed point (I) has $\tilde{g}=\nu_{B}=0$ and corresponds to two disconnected $Z_{2^{-}}$ symmetric theories. The potential $u_{k}\left(\tilde{\rho}_{1}, \tilde{\rho}_{2}\right)$ has minima at $\tilde{\rho}_{10}=\tilde{\rho}_{20}=\tilde{\rho}_{0} / 2$ (for positive and negative values of $\left.\phi_{1,2}\right)$.

c) The Cubic fixed point (C) has $\tilde{g}=3 \tilde{\lambda}, \nu_{B}=5 \nu_{A}$ and corresponds to two disconnected $Z_{2}$-symmetric theories, if the fields are redefined according to

$$
\phi_{1}^{\prime}=\frac{1}{\sqrt{2}}\left(\phi_{1}+\phi_{2}\right), \quad \phi_{2}^{\prime}=\frac{1}{\sqrt{2}}\left(\phi_{1}-\phi_{2}\right) .
$$

The potential $u_{k}\left(\tilde{\rho}_{1}, \tilde{\rho}_{2}\right)$ has minima at $\tilde{\rho}_{10}=\tilde{\rho}_{0}, \tilde{\rho}_{20}=0$ and $\tilde{\rho}_{20}=\tilde{\rho}_{0}, \tilde{\rho}_{10}=0$.

All these points are infrared unstable in the $\tilde{m}^{2}$ direction and are located on a critical surface $\tilde{m}_{c r}^{2}=\tilde{m}_{c r}^{2}\left(\tilde{\lambda}, \tilde{g}, \nu_{A}, \nu_{B}\right)<0$. Solutions of the evolution equations, which start above the critical surface, with $\tilde{m}^{2}>\tilde{m}_{c r}^{2}$, flow towards the region of positive $\tilde{m}^{2}$ for $t \rightarrow-\infty$, and correspond to theories in the symmetric phase. Solutions with $\tilde{m}^{2}<\tilde{m}_{c r}^{2}$ flow deep into the region of negative $\tilde{m}^{2}$ and correspond to theories in the phase with spontaneous symmetry breaking.

The relative stability of the fixed points on the critical surface determines which one governs the dynamics of the phase transition in the immediate vicinity of the critical temperature (or mass). It has been discussed in ref. [9], in a truncation that neglects derivatives of the potential higher than the second (and corresponds to $\nu_{A}, \nu_{B}=0$ ). All three fixed points are attractive in the $\tilde{\lambda}$ direction. However, the Ising and Cubic fixed points are repulsive in the $\tilde{g}$ direction, while the Heisenberg fixed point is totally attractive. There are four disconnected regions:

a) $3 \tilde{\lambda}>\tilde{g}>\tilde{\lambda}$ : The trajectories flow away from the Cubic towards the Heisenberg fixed point.

b) $\tilde{\lambda}>\tilde{g}>0$ : The trajectories flow away from the Ising towards the Heisenberg fixed point.

For parameters in the above two regions we expect second-order phase transitions, with critical dynamics governed by the three fixed points.

c) $\tilde{g}>3 \tilde{\lambda}$ : The trajectories flow away from the Cubic fixed point into a region of large $\tilde{g}$ and small $\tilde{\lambda}$. Eventually $\tilde{\lambda}$ turns negative at a finite value of $k$. At this point an instability arises which signals the presence of a first-order phase transition.

d) $\tilde{g}<0$ : The trajectories flow away from the Ising fixed point and cross the line $\tilde{g}=-\tilde{\lambda}$ at a finite $k$. This again implies the presence of an instability whose true nature is related to a first-order phase transition.

Flows that start on the lines $\tilde{g}=0, \tilde{\lambda}, 3 \tilde{\lambda}$ remain on these lines. No trajectories exist which connect the four regions $\tilde{g}>3 \tilde{\lambda}, 3 \tilde{\lambda}>\tilde{g}>\tilde{\lambda}, \tilde{\lambda}>\tilde{g}>0, \tilde{g}<0$.

We are interested in studying in detail the crossover from the Ising to the Heisenberg fixed point in the region $\tilde{\lambda}>\tilde{g}>0$. The region $3 \tilde{\lambda}>\tilde{g}>\tilde{\lambda}$ can be mapped on the region $\tilde{\lambda}>\tilde{g}>0$ through a redefinition of the fields [9] according to eqs. (3.9). This shows that 
the Ising and Cubic fixed points lead to an identical universal behaviour (and therefore to identical universal quantities, such as critical exponents and crossover curves).

\section{The crossover}

We would like to study the crossover from the Ising to the Heisenberg fixed point. In the vicinity of these fixed points the potential $u_{k}\left(\tilde{\rho}_{1}, \tilde{\rho}_{2}\right)$ has minima at $\tilde{\rho}_{10}=\tilde{\rho}_{20}=\tilde{\rho}_{0} / 2>0$ (for positive and negative values of $\phi_{1,2}$ ). In order to improve our quantitative accuracy in this regime (which we call the $\mathrm{M}$ regime), it is convenient to parametrize the potential in terms of its derivatives at the minimum. For this reason we define the parameters

$$
\begin{array}{cl}
\bar{\lambda}(k)=U_{11}\left(\rho_{0}\right)=U_{22}\left(\rho_{0}\right) & \bar{g}(k)=U_{12}\left(\rho_{0}\right) \\
\bar{\nu}_{A}(k)=U_{111}\left(\rho_{0}\right)=U_{222}\left(\rho_{0}\right) & \bar{\nu}_{B}(k)=U_{112}\left(\rho_{0}\right)=U_{122}\left(\rho_{0}\right),
\end{array}
$$

where $\rho_{10}=\rho_{20}=\rho_{0} / 2$ is the minimum of $U_{k}\left(\rho_{1}, \rho_{2}\right)$, related to the minimum of $u_{k}\left(\tilde{\rho}_{1}, \tilde{\rho}_{2}\right)$ through the first of eqs. (2.9). The equality of derivatives is imposed by the $(1 \leftrightarrow-1,2 \leftrightarrow$ $-2,1 \leftrightarrow 2)$ symmetry of the theory. The first derivatives $U_{1}\left(\rho_{0}\right)=U_{2}\left(\rho_{0}\right)$ are zero for non-zero $\rho_{0}$. Our approximation amounts to using for the potential the ansatz

$$
\begin{aligned}
U_{k}\left(\rho_{1}, \rho_{2}\right)= & \frac{\bar{\lambda}}{2}\left[\left(\rho_{1}-\frac{\rho_{0}}{2}\right)^{2}+\left(\rho_{2}-\frac{\rho_{0}}{2}\right)^{2}\right]+\bar{g}\left(\rho_{1}-\frac{\rho_{0}}{2}\right)\left(\rho_{2}-\frac{\rho_{0}}{2}\right) \\
& +\frac{\bar{\nu}_{A}}{6}\left[\left(\rho_{1}-\frac{\rho_{0}}{2}\right)^{3}+\left(\rho_{2}-\frac{\rho_{0}}{2}\right)^{3}\right] \\
& +\frac{\bar{\nu}_{B}}{2}\left[\left(\rho_{1}-\frac{\rho_{0}}{2}\right)^{2}\left(\rho_{2}-\frac{\rho_{0}}{2}\right)+\left(\rho_{1}-\frac{\rho_{0}}{2}\right)\left(\rho_{2}-\frac{\rho_{0}}{2}\right)^{2}\right] .
\end{aligned}
$$

We define dimensionless couplings according to eqs. (3.3) for the derivatives and

$$
\kappa(k)=Z_{k} k^{-1} \rho_{0}(k)
$$

for the minimum. The evolution equations for these couplings are lengthy and can be found in the appendix. They automatically preserve the discrete symmetry. The various fixed points are now located on a critical surface $\kappa_{c r}^{2}=\kappa_{c r}^{2}\left(\tilde{\lambda}, \tilde{g}, \nu_{A}, \nu_{B}\right)$, which is unstable in the $\kappa$ direction and separates the phase with spontaneous symmetry breaking from the symmetric one. Solutions of the evolution equation starting with $\kappa>\kappa_{c r}$ flow towards the region of large $\kappa$ and correspond to the phase with symmetry breaking. Eventually the minimum of the potential settles down at a finite value

$$
\rho_{0 R}=\lim _{k \rightarrow 0} Z_{k} \rho_{0}(k)=\lim _{k \rightarrow 0} k \kappa(k)
$$

Solutions starting with $\kappa<\kappa_{c r}$ lead to $\kappa(k)=0$ at some finite $k_{s}$. From this point on, the system is in the symmetric regime ( $\mathrm{S}$ regime) and we use a parametrization of

the potential according to eqs. (3.1) and (3.2), the rescaled variables defined in eqs. 
(3.3), and the evolution equations (3.4)-(3.8). Finally, if the evolution starts on the critical surface the flows never lead out of it. Instead they eventually approach the most attractive fixed point on the critical surface. This means that for $k \rightarrow 0$ the renormalized minimum of the potential is given by eq. (4.4) with finite $\kappa$. Therefore, $\rho_{0 R}=0$ and the critical surface corresponds to a second-order phase transition.

We numerically integrate the evolution equations by starting at some high momentum scale (ultraviolet cutoff) $k=\Lambda(t=0)$ where the potential $U_{\Lambda}$ is equal to the classical potential $V$. We consider $U_{\Lambda}$ quartic in the fields, and therefore given by eq. (4.2) with $\bar{\nu}_{A}(\Lambda)=\bar{\nu}_{B}(\Lambda)=0$. For the wave function renormalization we start with $Z_{\Lambda}=1$. We fine-tune $\rho_{0}(\Lambda) / \Lambda=\kappa(\Lambda)$, so that $\left|\delta \kappa_{\Lambda}\right|=\left|\kappa(\Lambda)-\kappa_{c r}\right| \ll 1$, and the system is very close to the critical surface. The subsequent evolution moves the parameters along the critical surface and close to various fixed points depending on their relative stability. We define the quantity

$$
x(k)=\frac{\bar{g}(k)}{\bar{\lambda}(k)}-1=\frac{\tilde{g}(k)}{\tilde{\lambda}(k)}-1
$$

in order to parametrize the distance from the Heisenberg fixed point. We have $x_{I}=-1$ at the Ising fixed point and $x_{H}=0$ at the Heisenberg one. We can arrange for the evolution to approach first the Ising fixed point by selecting $\delta x_{\Lambda}=x(\Lambda)+1 \ll 1$. Then $x(k)$ remains very close to its initial value while the running couplings approach their values at the Ising fixed point

$$
\kappa_{I}=8.57 \times 10^{-2}, \quad \tilde{\lambda}_{I}=8.05, \quad \tilde{g}_{I}=0, \quad\left(\nu_{A}\right)_{I}=75.8, \quad\left(\nu_{B}\right)_{I}=0 .
$$

Near the fixed point the evolution slows down, as the $\beta$-functions are almost zero. However, the Ising fixed point has two unstable directions and the system will eventually move away from it. One of these directions leads away from the critical surface, while the other is directed towards the Heisenberg fixed point. By selecting the starting point of the evolution so that $\delta x_{\Lambda} \gg\left|\delta \kappa_{\Lambda}\right|$, we can arrange for the flow to stay close to the critical surface while moving from one fixed point to the other. Eventually the running couplings approach their values at the Heisenberg fixed point

$$
\kappa_{H}=6.02 \times 10^{-2}, \quad \tilde{\lambda}_{H}=7.32, \quad \tilde{g}_{H}=7.32, \quad\left(\nu_{A}\right)_{H}=56.4, \quad\left(\nu_{B}\right)_{H}=56.4 .
$$

The trajectory in coupling space connecting the two fixed points is depicted in fig. 1, while the $\beta$-functions for the various couplings along the trajectory are plotted in fig. 2 . It is clear that the evolution is very slow near the fixed points where the $\beta$-functions are zero and fast in the middle of the trajectory. As long as $\left|\delta \kappa_{\Lambda}\right| / \delta x_{\Lambda} \ll 1$ the system stays close to the critical surface for a sufficiently long "time" $t$ for the Heisenberg fixed point to be reached. However, if the ratio $\left|\delta \kappa_{\Lambda}\right| / \delta x_{\Lambda}$ is increased the system leaves the critical surface at some earlier point of the evolution along the critical trajectory of fig. 1, and moves towards the phase with symmetry breaking or towards the symmetric one. For $\delta \kappa_{\Lambda}<0$ it runs into the $\mathrm{S}$ regime at some finite $k_{s}$ and eventually settles down in the symmetric phase with renormalized parameters [0, 9]

$$
m_{R}^{2}=\lim _{k \rightarrow 0} m^{2}(k)=\lim _{k \rightarrow 0} k^{2} \tilde{m}^{2}(k)
$$




$$
\begin{aligned}
& \lambda_{R}=\lim _{k \rightarrow 0} \lambda(k)=\lim _{k \rightarrow 0} k \tilde{\lambda}(k) \quad g_{R}=\lim _{k \rightarrow 0} g(k)=\lim _{k \rightarrow 0} k \tilde{g}(k) \quad x_{R}=\frac{g_{R}}{\lambda_{R}}-1 \\
& \nu_{A R}=\lim _{k \rightarrow 0} \nu_{A}(k) \quad \nu_{B R}=\lim _{k \rightarrow 0} \nu_{B}(k) .
\end{aligned}
$$

For $\delta \kappa_{\Lambda}>0$ the system settles down in the phase with symmetry breaking, with the minimum of the potential given by eq. (4.4) and the various couplings at the minimum defined in analogy with eqs. (4.8) 9 .

The singular behaviour of the theory in the critical region near the phase transition can be parametrized in terms of critical exponents. For example, the renormalized mass in the symmetric phase can be expressed as

$$
m_{R}^{2} \propto\left|\delta \kappa_{\Lambda}\right|^{2 \nu}
$$

The parameter $\nu$ can be computed as the slope of the function $\ln m_{R}^{2}=f\left(\ln \left|\delta \kappa_{\Lambda}\right|\right)$. If the system is in the vicinity of a fixed point, $\nu$ is constant and can be identified with the corresponding critical exponent. Moreover, this exponent can be related [1, 2, 20] to the most negative eigenvalue $\lambda_{1}$ of the matrix $\beta_{i, j}=\partial \beta_{i} / \partial a_{j}\left(\underset{\sim}{\text { with }} a_{j}=\kappa, \tilde{\lambda}, \tilde{g}, \nu_{A}, \nu_{B}\right)$, which describes the growth of small perturbations $\delta \kappa=\kappa-\kappa_{f p}, \delta \tilde{\lambda}=\tilde{\lambda}-\tilde{\lambda}_{f p}$, etc., around the fixed point, i.e.

$$
\nu=-\frac{1}{\lambda_{1}}
$$

In fig. 3 we plot the three smallest eigenvalues of the matrix $\beta_{i, j}$ for small perturbations $\delta \kappa=\kappa-\kappa_{t r}, \delta \tilde{\lambda}=\tilde{\lambda}-\tilde{\lambda}_{t r}$, etc., around the trajectory of fig. 1. For this calculation we employ the $\beta$-functions given in the appendix. We observe that the smallest eigenvalue remains negative along the whole trajectory. It corresponds to the unstable direction away from the critical surface. From its values at $x=-1$ and $x=0$, making use of eq. (4.10), we can infer the critical exponent $\nu$ of the Ising and Heisenberg models

$$
\begin{gathered}
\nu_{I}=0.700 \\
\nu_{H}=0.733 .
\end{gathered}
$$

The above values deviate at the $10 \%$ level from the more accurate results obtained through the $\epsilon$-expansion or summed perturbation series $\left(\nu_{I}=0.631(2), \nu_{H}=0.670(3)\right.$ [21]), or from results obtained with the same method and more extended truncations [7]. This is not surprising, as our very rough treatment of the wave function renormalization generates uncertainties of the order of the anomalous dimension $\eta$, as we mentioned at the end of section 2. We observe that the difference $\nu_{H}-\nu_{I}=0.033$ compares rather well with the high precision estimate 0.039 . We have verified the above values for the critical exponents by the explicit computation of the function $\ln m_{R}^{2}=f\left(\ln \left|\delta \kappa_{\Lambda}\right|\right)$. For this we integrated the evolution equations for theories with $\left|\delta \kappa_{\Lambda}\right|, \delta x_{\Lambda} \ll 1$, as we discussed in the previous paragraphs. For $\left|\delta \kappa_{\Lambda}\right| \gg \delta x_{\Lambda}$ the evolution approaches the Ising fixed point and slows

\footnotetext{
${ }^{5}$ In the vicinity of the Heisenberg fixed point the presence of a massless Goldstone mode results in zero values for $\lambda_{R}, g_{R}$ in the limit $k \rightarrow 0$. Quartic couplings can be defined at non-zero values of $k$ 12, 7 .
} 
down for a long "time" $t$. As a result the system loses memory of the initial conditions of the running (the classical parameters of the theory). Eventually the system leaves the critical surface in the vicinity of the Ising fixed point. The function $\ln m_{R}^{2}=f\left(\ln \left|\delta \kappa_{\Lambda}\right|\right)$ has a constant slope, which reproduces the value of eq. 4.11) for the exponent $\nu$. For theories with a smaller $\left|\delta \kappa_{\Lambda}\right| / \delta x_{\Lambda}$ ratio the evolution first approaches the Ising fixed point and then deviates from it along the critical surface. Finally it leaves the critical surface at some point along the trajectory of fig. 1 connecting the two fixed points. The function $\ln m_{R}^{2}=f\left(\ln \left|\delta \kappa_{\Lambda}\right|\right)$ now has a variable slope, since the growth of deviations from the critical surface depends on the average of the eigenvalue $\lambda_{1}$ along the part of the trajectory covered by the evolution. Only for $\left|\delta \kappa_{\Lambda}\right| \ll \delta x_{\Lambda}$ does the slope become constant again. For these theories the flow first moves from the Ising to the Heisenberg fixed point, and then spends a long "time" $t$ in its vicinity. During this "time" it loses memory of the previous evolution, before eventually leaving the critical surface. The calculation of the exponent $\nu$ reproduces the value of eq. (4.12). The error in the above values of $\nu$ originating in uncertainties of the numerical integration is less that $1 \%$ and, therefore, much smaller than the $10 \%$ error resulting from the truncations of the evolution equation.

We now turn to the second smallest eigenvalue of fig. 3, which changes sign along the trajectory connecting the two fixed points. Its negative sign at $x=-1$ indicates that the Ising fixed point has two unstable directions, while its positive value at $x=0$ indicates that the Heisenberg fixed point has only one. The eigenvalue $\lambda_{2}$ corresponds to the direction along the critical surface, which leads from the Ising to the more stable Heisenberg fixed point. Its value at $x=-1$ determines the growth of perturbations that couple the two disconnected $Z_{2}$-symmetric theories of the Ising fixed point. For the renormalized theory these perturbations are parametrized in terms of the dimensionless quantity $x_{R}+1=g_{R} / \lambda_{R}$. A crossover exponent $\varphi$ can be defined through the relation

$$
x_{R}+1 \propto\left|\delta \kappa_{\Lambda}\right|^{-\varphi} \text {. }
$$

In the vicinity of the Ising fixed point this exponent is related to the two smallest eigenvalues according to [1, 2, 20]

$$
\varphi=\frac{\lambda_{2}}{\lambda_{1}}=0.032
$$

Alternatively $\varphi$ can be computed from the slope of the function $\ln \left(x_{R}+1\right)=f\left(\ln \left|\delta \kappa_{\Lambda}\right|\right)$. The two results are in agreement. Comparison with more accurate results obtained through the $\epsilon$-expansion $(\varphi=0.110(5),[17,19])$ indicates that our approximations result in limited accuracy for such a small quantity. In fact, the eigenvalue $\left|\lambda_{2}\right|$ is accidentally very small on the scale of the other eigenvalues $\left|\lambda_{1}\right|$ and $\left|\lambda_{3}\right|$ (cf. fig. 3). A modest truncation error can easily change it by a factor 3 or 4 . It is not very difficult to increase the truncation level in order to reach a few per cent accuracy for $\nu$ [7] and correspondingly for $\varphi$. (We guess that a truncation at the level of ref. [7] should yield eigenvalues $\lambda_{i}$ with an accuracy of $\simeq 0.05$ and, therefore, an uncertainty in the $\varphi$ value $\simeq 0.03$.) A precise estimate of $\varphi$ is, however, not the purpose of this paper. As mentioned in the introduction we rather want to demonstrate that our method is capable of covering the whole crossover region between the two fixed points. 
In fig. 4 we plot the difference of the "effective" exponent $\nu=\partial \ln m_{R} / \partial \ln \left|\delta \kappa_{\Lambda}\right|$ from its value at the Ising fixed point as a function of $x(\Lambda)$. For this we integrate numerically the evolution equations by starting at the ultraviolet cutoff $k=\Lambda(t=0)$ where the potential $U_{\Lambda}$ is equal to the classical potential $V$. We consider $U_{\Lambda}$ quartic in the fields, with $\bar{\lambda}(\Lambda)=5$ and fine-tune $\kappa(\Lambda)$ so that the flows approach the critical surface. By varying $\left|\delta \kappa_{\Lambda}\right|$ we obtain theories with various values of the renormalized mass. In fig. 4 we also plot the renormalized parameter $x_{R}$ defined in eqs. (4.8). The curves (a) correspond to theories with renormalized mass $m_{R} / \Lambda \sim 10^{-4}-10^{-3}$, while the curves (b) correspond to theories with renormalized mass $m_{R} / \Lambda \sim 10^{-5}-10^{-4}$. The presence of small kinks in these curves gives a feeling of the typical errors resulting from the numerical integration - a less than $1 \%$ effect. It is clear that the evolution results in values for $x$ in the limit $k \rightarrow 0$ which are closer to the Heisenberg fixed point than the "initial value" $x(\Lambda)$. Both curves for $x_{R}$ are above the dotted line, which corresponds to $x_{R}=x(\Lambda)$. Theories with smaller renormalized mass stay close to the critical surface for longer "time" $t$, so that the attraction of the Heisenberg fixed point is more pronounced. For given $x(\Lambda)$ this results in $x_{R}$ being closer to zero for curve (b) than for curve (a). Similarly the value of $\nu$ for curve (b) is closer to the Heisenberg fixed point value than for curve (a). We have also included in fig. 4 the line corresponding to $-\lambda_{1}^{-1}(x)$ (dashed-dotted line), with $\lambda_{1}$ the most negative eigenvalue of the stability matrix plotted in fig. 3. For sufficiently small $m_{R} / \Lambda$ the flows quickly approach the critical trajectory of fig. 1 at some point $x \simeq x(\Lambda)$. Then the running follows the critical trajectory towards the Heisenberg fixed point, until eventually it moves away from the critical surface towards the symmetric regime. The resulting exponent $\nu$ corresponds to an average over the values of $-\lambda_{1}^{-1}(x)$ for the part of the flow close to the critical trajectory, along with some non-universal contributions from the initial and final running. For sufficiently small $m_{R} / \Lambda$ (such as for curve (b)) the latter contributions become insignificant. The resulting critical exponent $\nu$ is larger than the value of $-\lambda_{1}^{-1}$ at the point $x(\Lambda)$, because of the running towards the Heisenberg fixed point. For this reason the curve (b) for $\nu$ lies above the dashdotted line. For the curve (a) the non-universal initial and final running give significant contributions, which contaminate the universal part along the critical trajectory. The resulting "effective" exponent $\nu$ has a complicated dependence on $x(\Lambda)$. It should be pointed out that in most experimental situations the observed values of $m_{R} / \Lambda$ are not sufficiently small for the non-universal physics to be insignificant. It seems difficult to perform investigations of crossover based only on the value of the critical exponents. This would require to disentangle the crossover from the non-universal behaviour. Figure 4 is an explicit demonstration that our method can effectively deal with such situations. Starting from a given set of values for the parameters of the theory at the short distance scale $\Lambda$, both the universal and non-universal long distance physics can be determined through the integration of the evolution equations. 


\section{Conclusions}

We have demonstrated that the flow of the effective average action $\Gamma_{k}$ can be used for a description of crossover phenomena over the whole crossover region. We have computed both universal quantities - the eigenvalues $\lambda_{i}(x)$ of the stability matrix of small fluctuations along the crossover curve parametrized by $x$ - as well as actual "effective" exponents (see fig. 4) for given values of $m_{R} / \Lambda$. Even though an exact non-perturbative flow equation for $\Gamma_{k}$ is available, practical calculations are feasible only for truncations of $\Gamma_{k}$. These approximations have not been pushed to a high level of accuracy in the present paper. High precision calculations within the $\epsilon$-expansion have determined the crossover exponent $\varphi$ with very satisfactory accuracy in the past. However, for many practical applications the actually observed critical behaviour in crossover situations is not only determined by the universal critical behaviour. It is often a mixture of the flow towards the fixed points and the subsequent crossover flow from one fixed point to another. In these situations of "imperfect scaling", many standard methods, as for example the $\epsilon$ expansion, cannot be applied easily. The flow of the effective average action is directly formulated in three dimensions and includes the relevant degrees of freedom at every scale

$k$ (not only for $k$ in the scaling region). It can be used both in the symmetric phase and in the phase with spontaneous symmetry breaking, where the existence of massless Goldstone bosons hinders the application of many standard methods. For a given initial form of the "microscopic action" $\Gamma_{\Lambda}$, we can solve these equations numerically and compute the quantities of interest. We hope that the present method will develop in the future into a useful practical tool for quantitative investigations of crossover problems. 


\section{Appendix: The evolution equations in the $M$ regime}

It is easier to derive the evolution equations for the dimensionless derivatives in eqs. (3.3) and the rescaled minimum of the potential of eqs. (4.3) by making use of the evolution equation (2.10) for the rescaled potential $u_{k}\left(\tilde{\rho}_{1}, \tilde{\rho}_{2}\right)$ in three dimensions

$$
\begin{aligned}
\frac{\partial}{\partial t} u\left(\tilde{\rho}_{1}, \tilde{\rho}_{2}\right) & =-3 u+(1+\eta)\left(\tilde{\rho}_{1} u_{1}+\tilde{\rho}_{2} u_{2}\right)+\zeta \\
\zeta & =-v_{3} L_{0}^{3}\left(\tilde{m}_{1}^{2}\right)-v_{3} L_{0}^{3}\left(\tilde{m}_{2}^{2}\right),
\end{aligned}
$$

where the rescaled mass eigenvalues are given by

$$
\begin{aligned}
\tilde{m}_{1,2}^{2}\left(\tilde{\rho}_{1}, \tilde{\rho}_{2}\right) & =\frac{1}{2}\left(u_{1}+u_{2}+2 u_{11} \tilde{\rho}_{1}+2 u_{22} \tilde{\rho}_{2} \pm A\right) \\
A & =\left[\left(u_{1}-u_{2}+2 u_{11} \tilde{\rho}_{1}-2 u_{22} \tilde{\rho}_{2}\right)^{2}+16 u_{12}^{2} \tilde{\rho}_{1} \tilde{\rho}_{2}\right]^{\frac{1}{2}} .
\end{aligned}
$$

The evolution of $\kappa(k)$ is obtained by taking the total $t$-derivative of the minimization conditions $\partial u_{k} /\left.\partial \tilde{\rho}_{1}\right|_{\kappa}=\partial u_{k} /\left.\partial \tilde{\rho}_{2}\right|_{\kappa}=0$. We obtain

$$
\frac{d \kappa}{d t}=-(1+\eta) \kappa-\left.\frac{2}{\tilde{\lambda}+\tilde{g}} \frac{\partial \zeta}{\partial \tilde{\rho}_{1}}\right|_{\kappa}
$$

For the derivatives we find

$$
\begin{aligned}
\frac{d \tilde{\lambda}}{d t} & =(-1+2 \eta) \tilde{\lambda}+\left.\frac{\partial^{2} \zeta}{\partial \tilde{\rho}_{1}^{2}}\right|_{\kappa}-\left.\frac{\nu_{A}+\nu_{B}}{\tilde{\lambda}+\tilde{g}} \frac{\partial \zeta}{\partial \tilde{\rho}_{1}}\right|_{\kappa} \\
\frac{d \tilde{g}}{d t} & =(-1+2 \eta) \tilde{g}+\left.\frac{\partial^{2} \zeta}{\partial \tilde{\rho}_{1} \partial \tilde{\rho}_{2}}\right|_{\kappa}-\left.\frac{2 \nu_{B}}{\tilde{\lambda}+\tilde{g}} \frac{\partial \zeta}{\partial \tilde{\rho}_{1}}\right|_{\kappa} \\
\frac{d \nu_{A}}{d t} & =3 \eta \nu_{A}+\left.\frac{\partial^{3} \zeta}{\partial \tilde{\rho}_{1}^{3}}\right|_{\kappa} \\
\frac{d \nu_{B}}{d t} & =3 \eta \nu_{B}+\left.\frac{\partial^{3} \zeta}{\partial \tilde{\rho}_{1}^{2} \partial \tilde{\rho}_{2}}\right|_{\kappa} .
\end{aligned}
$$

The necessary $\tilde{\rho}$-derivatives of $\zeta$ are given by

$$
\begin{aligned}
\frac{\partial \zeta}{\partial \tilde{\rho}_{1}}= & v_{3}\left[\frac{\partial \tilde{m}_{1}^{2}}{\partial \tilde{\rho}_{1}} L_{1}^{3}\left(\tilde{m}_{1}^{2}\right)+\frac{\partial \tilde{m}_{2}^{2}}{\partial \tilde{\rho}_{1}} L_{1}^{3}\left(\tilde{m}_{2}^{2}\right)\right] \\
\frac{\partial^{2} \zeta}{\partial \tilde{\rho}_{1}^{2}}= & -v_{3}\left\{\left[\frac{\partial \tilde{m}_{1}^{2}}{\partial \tilde{\rho}_{1}}\right]^{2} L_{2}^{3}\left(\tilde{m}_{1}^{2}\right)+\left[\frac{\partial \tilde{m}_{2}^{2}}{\partial \tilde{\rho}_{1}}\right]^{2} L_{2}^{3}\left(\tilde{m}_{2}^{2}\right)\right\} \\
& +v_{3}\left\{\frac{\partial^{2} \tilde{m}_{1}^{2}}{\partial \tilde{\rho}_{1}^{2}} L_{1}^{3}\left(\tilde{m}_{1}^{2}\right)+\frac{\partial^{2} \tilde{m}_{2}^{2}}{\partial \tilde{\rho}_{1}^{2}} L_{1}^{3}\left(\tilde{m}_{2}^{2}\right)\right\} \\
\frac{\partial^{2} \zeta}{\partial \tilde{\rho}_{1} \partial \tilde{\rho}_{2}}= & -v_{3}\left\{\frac{\partial \tilde{m}_{1}^{2}}{\partial \tilde{\rho}_{1}} \frac{\partial \tilde{m}_{1}^{2}}{\partial \tilde{\rho}_{2}} L_{2}^{3}\left(\tilde{m}_{1}^{2}\right)+\frac{\partial \tilde{m}_{2}^{2}}{\partial \tilde{\rho}_{1}} \frac{\partial \tilde{m}_{2}^{2}}{\partial \tilde{\rho}_{2}} L_{2}^{3}\left(\tilde{m}_{2}^{2}\right)\right\}
\end{aligned}
$$




$$
\begin{aligned}
& +v_{3}\left\{\frac{\partial^{2} \tilde{m}_{1}^{2}}{\partial \tilde{\rho}_{1} \partial \tilde{\rho}_{2}} L_{1}^{3}\left(\tilde{m}_{1}^{2}\right)+\frac{\partial^{2} \tilde{m}_{2}^{2}}{\partial \tilde{\rho}_{1} \partial \tilde{\rho}_{2}} L_{1}^{3}\left(\tilde{m}_{2}^{2}\right)\right\} \\
\frac{\partial^{3} \zeta}{\partial \tilde{\rho}_{1}^{3}}= & 2 v_{3}\left\{\left[\frac{\partial \tilde{m}_{1}^{2}}{\partial \tilde{\rho}_{1}}\right]^{3} L_{3}^{3}\left(\tilde{m}_{1}^{2}\right)+\left[\frac{\partial \tilde{m}_{2}^{2}}{\partial \tilde{\rho}_{1}}\right]^{3} L_{3}^{3}\left(\tilde{m}_{2}^{2}\right)\right\} \\
& -3 v_{3}\left\{\frac{\partial \tilde{m}_{1}^{2}}{\partial \tilde{\rho}_{1}} \frac{\partial^{2} \tilde{m}_{1}^{2}}{\partial \tilde{\rho}_{1}^{2}} L_{2}^{3}\left(\tilde{m}_{1}^{2}\right)+\frac{\partial \tilde{m}_{2}^{2}}{\partial \tilde{\rho}_{1}} \frac{\partial^{2} \tilde{m}_{2}^{2}}{\partial \tilde{\rho}_{1}^{2}} L_{2}^{3}\left(\tilde{m}_{2}^{2}\right)\right\} \\
\frac{\partial^{3} \zeta}{\partial \tilde{\rho}_{1}^{2} \partial \tilde{\rho}_{2}}= & 2 v_{3}\left\{\left[\frac{\partial \tilde{m}_{1}^{2}}{\partial \tilde{\rho}_{1}}\right]^{2} \frac{\partial \tilde{m}_{1}^{2}}{\partial \tilde{\rho}_{2}} L_{3}^{3}\left(\tilde{m}_{1}^{2}\right)+\left[\frac{\partial \tilde{m}_{2}^{2}}{\partial \tilde{\rho}_{1}}\right]^{2} \frac{\partial \tilde{m}_{2}^{2}}{\partial \tilde{\rho}_{2}} L_{3}^{3}\left(\tilde{m}_{2}^{2}\right)\right\} \\
& -2 v_{3}\left\{\frac{\left.\left.\partial \tilde{m}_{1}^{2}\right)+\frac{\partial^{3} \tilde{m}_{2}^{2}}{\partial \tilde{\rho}_{1}} L_{1}^{3}\left(\tilde{m}_{2}^{2}\right)\right\}}{\partial \tilde{\rho}_{1} \partial \tilde{\rho}_{2}} L_{2}^{3}\left(\tilde{m}_{1}^{2}\right)+\frac{\partial \tilde{m}_{2}^{2}}{\partial \tilde{\rho}_{1}} \frac{\partial^{2} \tilde{m}_{2}^{2}}{\partial \tilde{\rho}_{1} \partial \tilde{\rho}_{2}} L_{2}^{3}\left(\tilde{m}_{2}^{2}\right)\right\} \\
& -v_{3}\left\{\frac{\partial^{2} \tilde{m}_{1}^{2}}{\partial \tilde{\rho}_{1}^{2}} \frac{\partial \tilde{m}_{1}^{2}}{\partial \tilde{\rho}_{2}} L_{2}^{3}\left(\tilde{m}_{1}^{2}\right)+\frac{\partial^{2} \tilde{m}_{2}^{2}}{\partial \tilde{\rho}_{1}^{2}} \frac{\partial \tilde{m}_{2}^{2}}{\partial \tilde{\rho}_{2}} L_{2}^{3}\left(\tilde{m}_{2}^{2}\right)\right\} \\
& +v_{3}\left\{\frac{\partial^{3} \tilde{m}_{1}^{2}}{\partial \tilde{\rho}_{1}^{2} \partial \tilde{\rho}_{2}} L_{1}^{3}\left(\tilde{m}_{1}^{2}\right)+\frac{\partial^{3} \tilde{m}_{2}^{2}}{\partial \tilde{\rho}_{1}^{2} \partial \tilde{\rho}_{2}} L_{1}^{3}\left(\tilde{m}_{2}^{2}\right)\right\} \cdot
\end{aligned}
$$

At the minimum the mass eigenvalues of eqs. (A.2) and their derivatives are given by

$$
\begin{aligned}
& \tilde{m}_{1,2}^{2}=(\tilde{\lambda} \pm \tilde{g}) \kappa \\
& \left.\frac{\partial \tilde{m}_{1,2}^{2}}{\partial \tilde{\rho}_{1}}\right|_{\kappa}=\left.\frac{\partial^{2} \tilde{m}_{1,2}^{2}}{\partial \tilde{\rho}_{1}^{2}}\right|_{\kappa}=\frac{1}{2}\left[3 \tilde{\lambda}+\tilde{g}+\left(\nu_{A}+\nu_{B}\right) \kappa \pm\left.\frac{\partial A}{\partial \tilde{\rho}_{1}}\right|_{\kappa}\right] \\
& \left.\frac{\partial^{2} \tilde{m}_{1,2}^{2}}{\partial \tilde{\rho}_{1}^{2}}\right|_{\kappa}=\frac{1}{2}\left(5 \nu_{A}+\nu_{B} \pm\left.\frac{\partial^{2} A}{\partial \tilde{\rho}_{1}^{2}}\right|_{\kappa}\right) \\
& \left.\frac{\partial^{2} \tilde{m}_{1,2}^{2}}{\partial \tilde{\rho}_{1} \partial \tilde{\rho}_{2}}\right|_{\kappa}=\frac{1}{2}\left(6 \nu_{B} \pm\left.\frac{\partial^{2} A}{\partial \tilde{\rho}_{1} \partial \tilde{\rho}_{2}}\right|_{\kappa}\right) \\
& \left.\frac{\partial^{3} \tilde{m}_{1,2}^{2}}{\partial \tilde{\rho}_{1}^{3}}\right|_{\kappa}= \pm\left.\frac{1}{2} \frac{\partial^{3} A}{\partial \tilde{\rho}_{1}^{3}}\right|_{\kappa} \\
& \left.\frac{\partial^{3} \tilde{m}_{1,2}^{2}}{\partial \tilde{\rho}_{1}^{2} \partial \tilde{\rho}_{2}}\right|_{\kappa}= \pm\left.\frac{1}{2} \frac{\partial^{3} A}{\partial \tilde{\rho}_{1}^{2} \partial \tilde{\rho}_{2}}\right|_{\kappa},
\end{aligned}
$$

with

$$
\begin{aligned}
A & =2 \tilde{g} \kappa \\
\left.\frac{\partial A}{\partial \tilde{\rho}_{1}}\right|_{\kappa} & =\frac{1}{2 A}\left(8 \tilde{g} \nu_{B} \kappa^{2}+8 \tilde{g}^{2} \kappa\right)
\end{aligned}
$$




$$
\begin{aligned}
&\left.\frac{\partial^{2} A}{\partial \tilde{\rho}_{1}^{2}}\right|_{\kappa}=-\frac{1}{A}\left(\left.\frac{\partial A}{\partial \tilde{\rho}_{1}}\right|_{\kappa}\right)^{2}+\frac{1}{2 A} B \\
& B=2\left[3 \tilde{\lambda}-\tilde{g}+\left(\nu_{A}-\nu_{B}\right) \kappa\right]^{2}+8 \nu_{B}^{2} \kappa^{2}+32 \tilde{g} \nu_{B} \kappa \\
&\left.\frac{\partial^{2} A}{\partial \tilde{\rho}_{1} \partial \tilde{\rho}_{2}}\right|_{\kappa}=-\frac{1}{A}\left(\left.\frac{\partial A}{\partial \tilde{\rho}_{1}}\right|_{\kappa}\right)^{2}+\frac{1}{2 A} D \\
& D=-2\left[3 \tilde{\lambda}-\tilde{g}+\left(\nu_{A}-\nu_{B}\right) \kappa\right]^{2}+8 \nu_{B}^{2} \kappa^{2}+32 \tilde{g} \nu_{B} \kappa+16 \tilde{g}^{2} \\
&\left.\frac{\partial^{3} A}{\partial \tilde{\rho}_{1}^{3}}\right|_{\kappa}=\frac{1}{A^{2}}\left(\left.\frac{\partial A}{\partial \tilde{\rho}_{1}}\right|_{\kappa}\right)^{3}-\frac{2}{A}\left(\left.\frac{\partial A}{\partial \tilde{\rho}_{1}}\right|_{\kappa}\right)\left(\left.\frac{\partial^{2} A}{\partial \tilde{\rho}_{1}^{2}}\right|_{\kappa}\right)-\frac{1}{2 A^{2}}\left(\left.\frac{\partial A}{\partial \tilde{\rho}_{1}}\right|_{\kappa}\right) B+\frac{1}{2 A} B_{1} \\
& B_{1}=6\left(5 \nu_{A}-\nu_{B}\right)\left[3 \tilde{\lambda}-\tilde{g}+\left(\nu_{A}-\nu_{B}\right) \kappa\right]+48 \nu_{B}^{2} \kappa \\
&\left.\frac{\partial^{3} A}{\partial \tilde{\rho}_{1}^{2} \partial \tilde{\rho}_{2}}\right|_{\kappa}=\frac{1}{A^{2}}\left(\left.\frac{\partial A}{\partial \tilde{\rho}_{1}}\right|_{\kappa}\right)^{3}-\frac{2}{A}\left(\left.\frac{\partial A}{\partial \tilde{\rho}_{1}}\right|_{\kappa}\right)\left(\left.\frac{\partial^{2} A}{\partial \tilde{\rho}_{1} \partial \tilde{\rho}_{2}}\right|_{\kappa}\right)-\frac{1}{2 A^{2}}\left(\left.\frac{\partial A}{\partial \tilde{\rho}_{1}}\right|_{\kappa}\right) B+\frac{1}{2 A} B_{2} \\
& B_{2}=-2\left(5 \nu_{A}-\nu_{B}\right)\left[3 \tilde{\lambda}-\tilde{g}+\left(\nu_{A}-\nu_{B}\right) \kappa\right]+48 \nu_{B}^{2} \kappa+64 \tilde{g} \nu_{B} \cdot
\end{aligned}
$$




\section{References}

[1] K.G. Wilson and I.G. Kogut, Phys. Rep. 12, 75 (1974).

[2] F.J. Wegner, in: Phase Transitions and Critical Phenomena, vol. 6, eds. C. Domb and M.S. Greene, Academic Press (1976).

[3] F.J. Wegner and A. Houghton, Phys. Rev. A 8, 401 (1973); S. Weinberg, Critical phenomena for field theorists, in Erice Subnuc. Phys. 1 (1976); J.F. Nicoll and T.S. Chang, Phys. Lett. A 62, 287 (1977); T.S. Chang, J.F. Nicoll and J.E. Young, Phys. Lett. A 67, 287 (1978); J. Polchinski, Nucl. Phys. B 231, 269 (1984); A. Hasenfratz and P. Hasenfratz, Nucl. Phys. B 270, 687 (1986); T.S. Chang, D.D. Vvedensky and J.F. Nicoll, Phys. Rep. 217, 279 (1992).

[4] C. Wetterich, Phys. Lett. B 301, 90 (1993).

[5] J. Langer, Ann. Phys. 41, 108 (1967); ibid. 54, 258 (1969); Physica 73, 61 (1974).

[6] C. Wetterich, Z. Phys. C 57, 451 (1993).

[7] N. Tetradis and C. Wetterich, Nucl Phys. B 422, 541 (1994).

[8] S. Bornholdt, N. Tetradis and C. Wetterich, Phys. Lett. B 348, 89 (1995).

[9] S. Bornholdt, N. Tetradis and C. Wetterich, preprint HD-THEP-94-28 and OUTP 95-02 P, to appear in Phys. Rev. D.

[10] J. Adams, J. Berges, S. Bornholdt, F. Freire, N. Tetradis and C. Wetterich, Mod. Phys. Lett. A 10, 2367 (1995).

[11] J. Berges, N. Tetradis and C. Wetterich, preprint HD-THEP-95-27 and OUTP 95$27 \mathrm{P}$.

[12] N. Tetradis and C. Wetterich, Nucl. Phys. B 398, 659 (1993).

[13] M. Reuter, N. Tetradis and C. Wetterich, Nucl. Phys. B 401, 567 (1993).

[14] N. Tetradis and C. Wetterich, Int. J. Mod. Phys. A 9, 4029 (1994).

[15] N. Tetradis and C. Wetterich, Nucl. Phys. B 383, 197 (1992).

[16] T.R. Morris, Phys. Lett. B 334, 355 (1994).

[17] A. Aharony, in: Phase Transitions and Critical Phenomena, vol. 6, eds. C. Domb and M.S. Greene, Academic Press (1976).

[18] J. Rudnick, Phys. Rev. B 18, 1406 (1978).

[19] D.J. Amit, Field Theory, the Renormalization Group, and Critical Phenomena, World Scientific (1984).

[20] N. Goldenfeld, Lectures on Phase Transitions and the Renormalization Group, Addison-Wesley (1992).

[21] J. Zinn-Justin, Quantum Field Theory and Critical Phenomena, Oxford Science Publications (1989). 


\section{Figures}

Fig. 1 : The trajectory on the critical surface which leads from the Ising to the Heisenberg fixed point.

Fig. 2 : The $\beta$-functions for the various couplings along the trajectory of fig. 1.

Fig. 3 : The three smallest eigenvalues for perturbations around the trajectory of fig. 1.

Fig. 4 : The difference $\beta-\beta_{I}$ and the renormalized parameter $x_{R}$ as a function of the value of $x$ at the cutoff. Lines (a) correspond to theories with renormalized mass $m_{R} / \Lambda \sim 10^{-4}-10^{-3}$, while lines (b) correspond to theories with renormalized mass $m_{R} / \Lambda \sim 10^{-5}-10^{-4}$. 


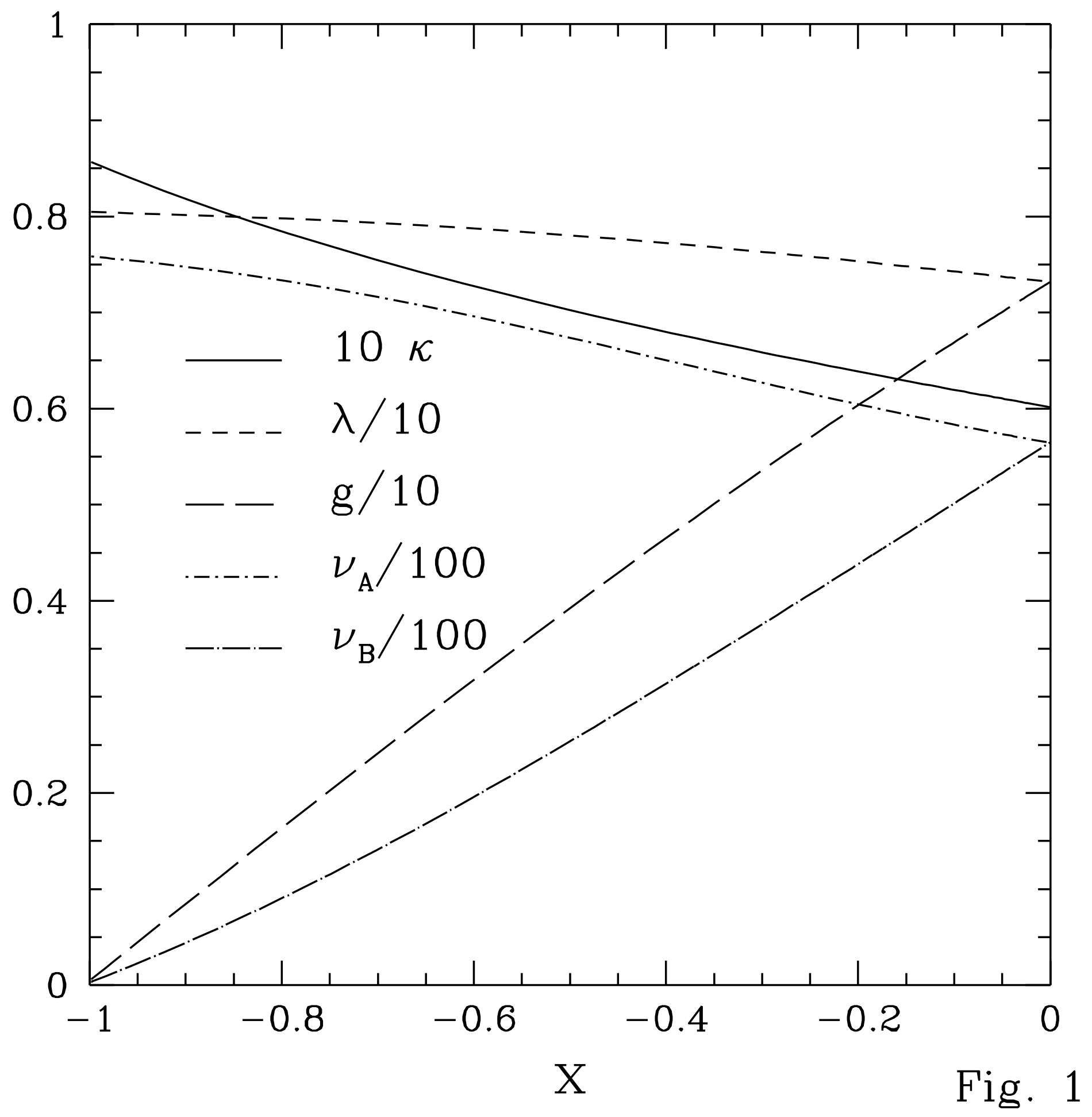




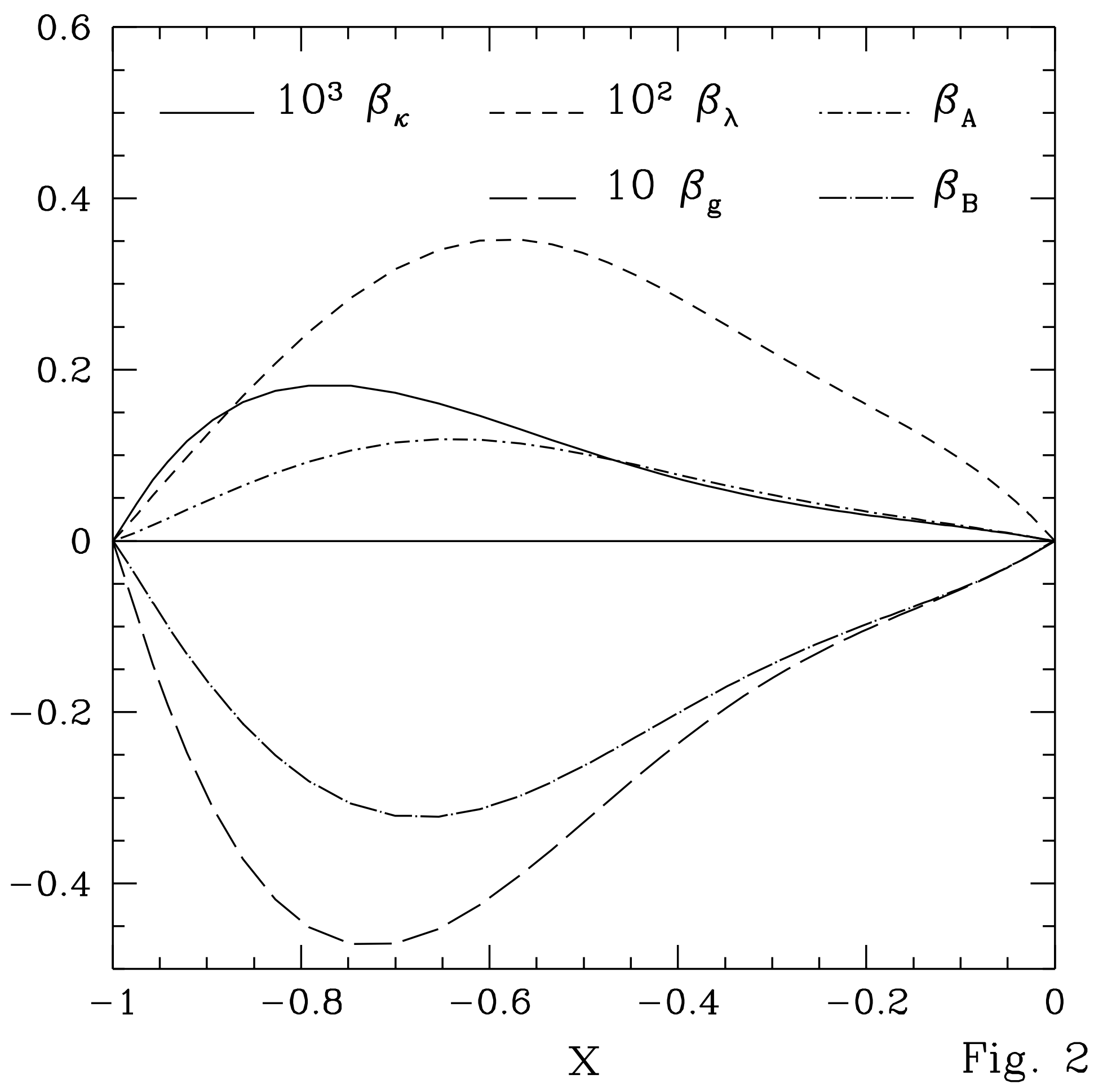




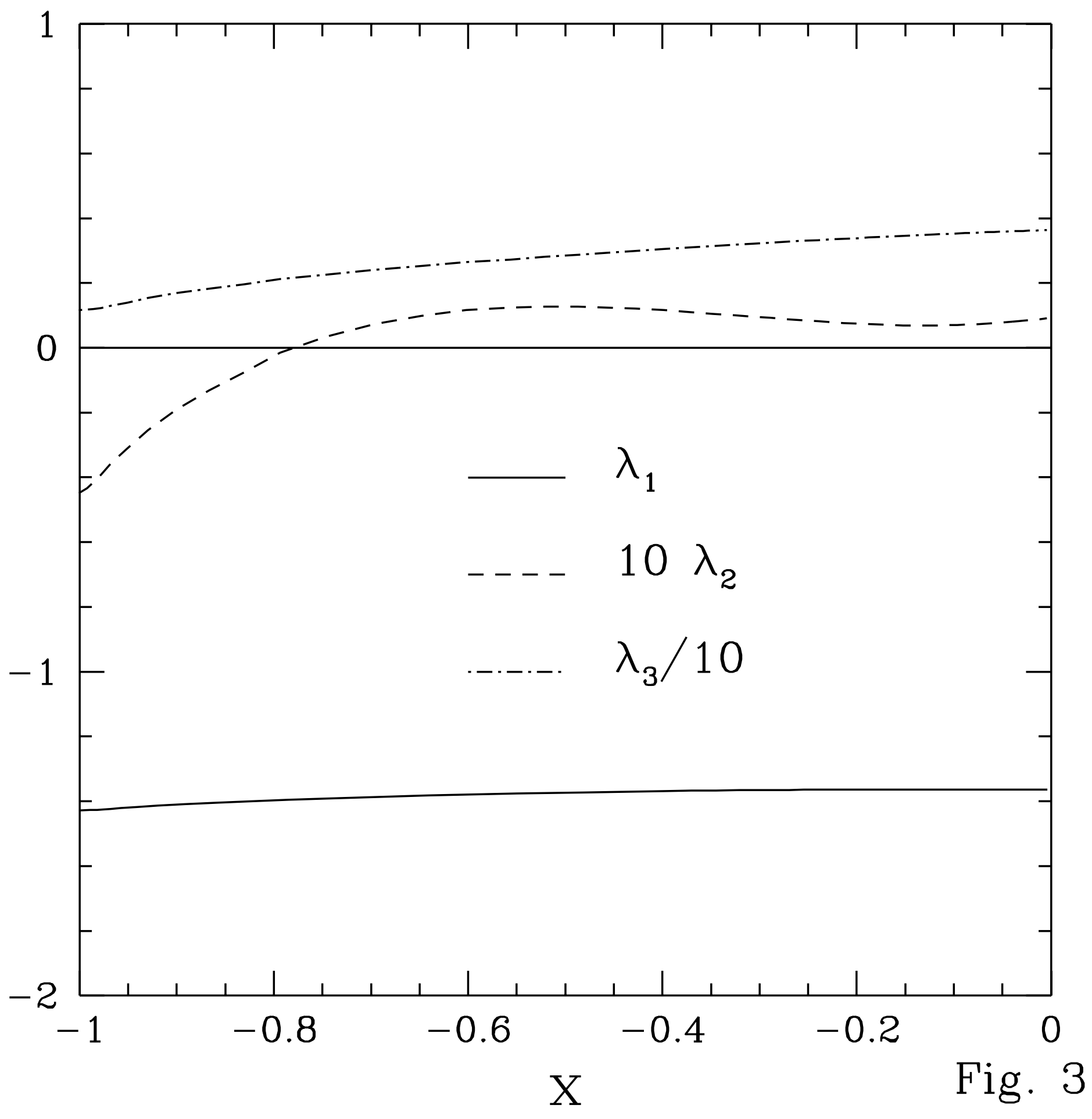




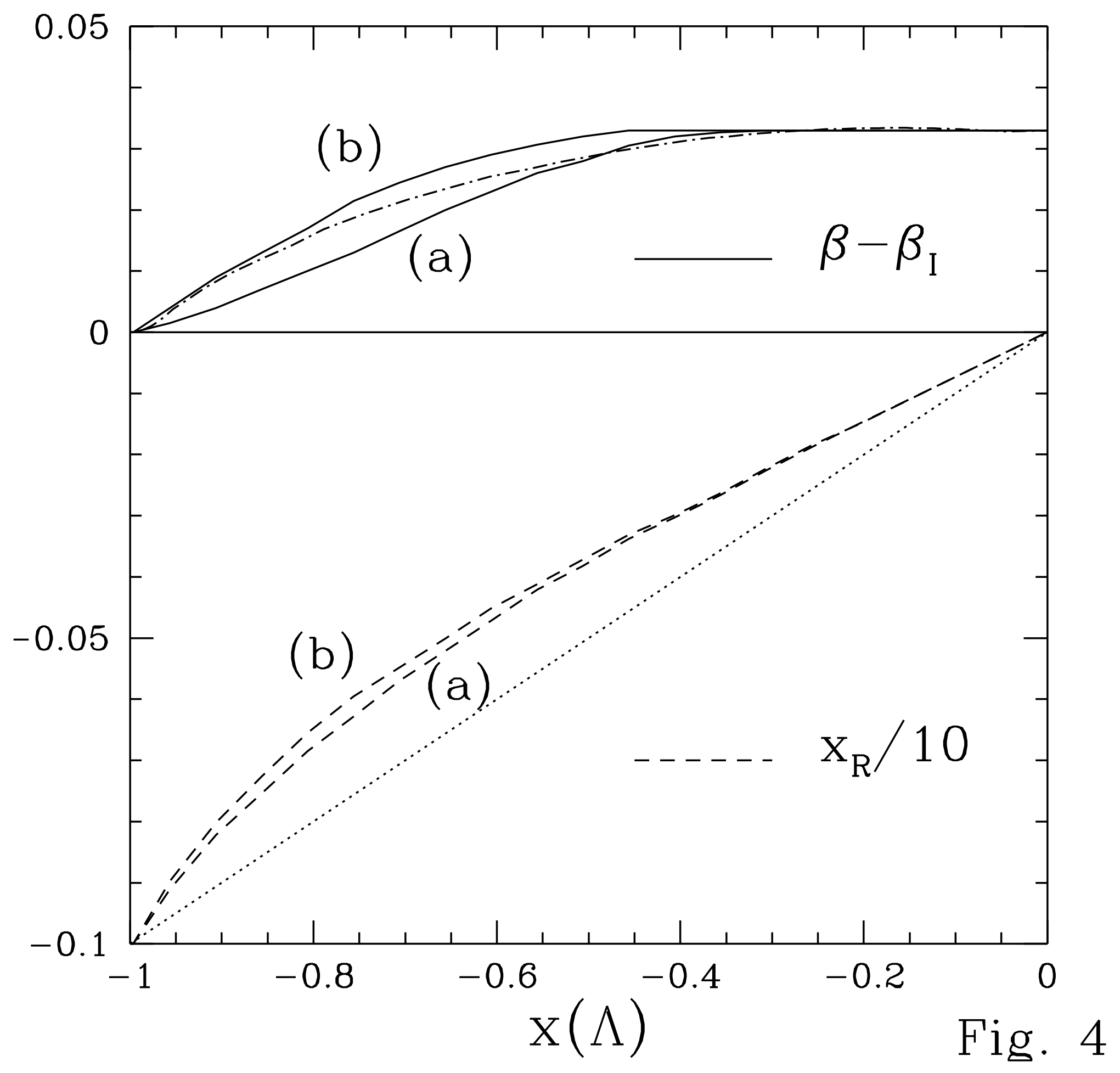

